\title{
Antibacterial Properties of Silk Fibroin/Chitosan Blend Films Loaded with Plant Extract
}

\author{
Guldemet Basal*, Duygu Altıok $^{1}$, and Oguz Bayraktar ${ }^{1}$ \\ Department of Textile Engineering, Ege University, Bornova-Izmir, Turkey \\ ${ }^{1}$ Department of Chemical Engineering, Izmir Institute of Technology, Urla-Izmir, Turkey
}

(Received May 3, 2009; Accepted December 7, 2009)

\begin{abstract}
The silk fibroin (SF)/chitosan (CHI) blend films were prepared by dissolving them in formic acid. The morphology of the films was examined by scanning electron microscopy (SEM). The roughness of the membranes was determined by atomic force microscopy (AFM). These films were treated with the extracts of Pistacia terebinthus, Pistacia lentiscus, and Hypericum empetrifolium. Folin-Ciocalteu assay was used to determine the amount of total phenols adsorbed on these blend films. The antibacterial properties of films were tested using disc diffusion and turbidity measurement methods against Escherichia coli and Staphylococcus epidermidis. The release of adsorbed phenolics from the film surface resulted in antibacterial properties.
\end{abstract}

Keywords: Silk fibroin, Chitosan, Antibacterial, P. terebinthus, P. lentiscus, H. empetrifolium

\section{Introduction}

Silk has been commercially used as surgical sutures for decades and as a source of textile-grade fibers for centuries [1]. Silk synthesized by Bombyx mori consists of two kinds of proteins, sericin and fibroin. Recently, interest has been concentrating and dramatically increasing on the use of several processed forms of the solubilized silk fibroin in (bio)technological materials and biomedical applications [1-9]. Silk fibroin is an interesting starting material for developing different forms of materials (e.g. gels, powder, foams, films, etc) [10]. Silk fibroin film has good oxygen permeability in its wet state, similar to that of human skin, which suggests the promising applications of silk fibroin as a wound dressing [11,12]. However, the SF film is too brittle to be used in biomedical applications. Due to this reason it is usually used by blending with other natural polymers such as chitosan [13]. Chitosan is widely used in the cosmetic and food industries and in medical textiles as wound dressing biomaterial [14].

An ideal dressing should maintain a moist environment at the wound interface, allow gaseous exchange, act as a barrier to microorganisms, and remove excess exudates. It is critical that the wound contact layer should include compounds which accelerate the healing. With the addition of antibiotics and antibacterial components to this layer, the wound dressing attains antimicrobial characteristics and the growth of infection-causing bacteria is prevented to a large extent [15]. The previous studies revealed that in addition to antimicrobial compounds, the use of antioxidant compounds in wound dressings speeds up the healing of chronic wounds [16]. It has been known for years that several plant extracts which contain natural compounds with different biologic

*Corresponding author: guldemet.basal@ege.edu.tr activities such as antioxidant, antimicrobial activities have wound healing effect [17].

Although several researchers investigated the conformations and mechanical properties of the SF/CHI blend films [8,11], some important characteristics of the plant extract loaded blend films have not been reported yet. The antibacterial properties of these films should satisfy the basic requirements for medical textile applications such as wound dressings.

The main objective of this study was to investigate the antibacterial properties of silk fibroin and chitosan blend films loaded with phenolics of Pistacia terebinthus (PT), Pistacia lentiscus (PL), and Hypericum empetrifolium (HE) extracts having both antioxidant and antimicrobial activities.

\section{Experimental}

\section{Materials}

Silk fibroin was obtained in reeled form from Bursa Institute for Silkworm Research (Bursa, Turkey). The chitosan was purchased from Aldrich Company. Ethanol (absolute GR for analysis) was obtained from Merck (Darmstadt, Germany). Deionized water was used during all experiments.

\section{Preparation of Plant Extracts}

Plant species ( $P$. terebinthus, $P$. lentiscus, and $H$. empetrifolium) used in this study were collected from Karaburun region in Izmir/Turkey. The air dried plant materials were ground in a blender with a particular size to ensure the plant powders in identical size. Ten gram of each plant powder was extracted for $2 \mathrm{~h}$ with $200 \mathrm{~m} l$ of $80 \%$ (v/ v) aqueous ethanol at $35{ }^{\circ} \mathrm{C}, 180 \mathrm{rpm}$ by using a thermoshaker. Then the samples were centrifuged at $5000 \mathrm{rpm}$ and the supernatant was evaporated with a rotary evaporator to remove ethanol. The remaining aqueous solutions were 
lyophilized to obtain crude extracts.

\section{Preparation of SF/CHI Blend Films}

In order to assure complete removal of sericin; silk was processed three times in boiling $\left(98-100{ }^{\circ} \mathrm{C}\right) 0.5 \mathrm{wt} \%$ $\mathrm{Na}_{2} \mathrm{CO}_{3}$ solution for $1 \mathrm{~h}$. The $\mathrm{SF} / \mathrm{CHI}$ blend films were prepared by using the stock solutions of $1.5 \%(\mathrm{w} / \mathrm{v}) \mathrm{SF}$ and $3 \%(\mathrm{w} / \mathrm{v}) \mathrm{CHI}$ dissolved in formic acid. The solutions were blended in the ratios of 75/25, 50/50, and 25/75 of SF/CHI. The blends were cast in plastic Petri dishes with a diameter of $60 \mathrm{~mm}$ and dried at room temperature over 2 days. The controls were $100 \% \mathrm{SF}$ and $100 \% \mathrm{CHI}$. The total weight of the solutions to be poured into each Petri dish was kept constant at $0.3 \mathrm{~g}$. The resulting films were about $0.10 \mathrm{~mm}$ in average thickness. Discs having a diameter of $10 \mathrm{~mm}$ were cut from the prepared films for plant extract adsorption experiments and antibacterial tests.

\section{Characterization of SF/CHI Blend Films}

The morphology of the surfaces of the SF/CHI films was examined by scanning electron microscopy (SEM, Philips, XL-30S FG). The roughness of the membranes was determined by atomic force microscopy (AFM, Digital Instruments MMSPM). The contact mode was used and $10 \mu \mathrm{m}$ scales were used for the area scanned.

The tensile strength and percent of elongation were measured under dry state of films using a Shimadzu Universal Testing Machine (AGI-10K, compression and tension). The experimental conditions were $3-\mathrm{N}$ initial load cell, $2 \mathrm{~mm} / \mathrm{min}$ tensile speed, and $16 \times 60 \times 0.10 \mathrm{~mm}$ film strip dimensions. The load to the film to break was plotted as a function of extension of clamps, then the data was converted to the corresponding tensile stress-tensile strain curve. Only, the values of breaking strength of films were determined to make a relative comparison between the films prepared at different blend ratios based on their properties of adsorbing phenolics from plant extracts.

\section{Preparation of Plant Extract Loaded SF/CHI Films}

The blend films made of SF and CHI were cut into discs having a diameter of $10 \mathrm{~mm}$. Approximately $1 \mathrm{~g}$ of extracts of PT, PL, and HE were dissolved in $25 \mathrm{~m} l$ of $70 \%$ aqueous ethanol solution. $2 \mathrm{~m} l$ of this plant extract solution was added into each well of a 12 well-plate containing about $0.050 \mathrm{~g}$ of film discs. Then the plates were placed in a thermo-shaker $\left(25^{\circ} \mathrm{C}, 90 \mathrm{rpm}\right.$, and $\left.6 \mathrm{~h}\right)$ for the adsorption of phenolics on the surface of film discs. After adsorption experiments total phenol contents in each well was measured to determine the amount of phenols adsorbed by the film discs.

\section{Determination of Total Phenol Contents}

Total phenol content of plant extracts was determined by using Folin-ciocalteu method. $100 \mu l$ plant extract solutions (before and after adsorption experiments) or standard (Gallic acid) solutions were mixed with $2.5 \mathrm{~m} l$ Folin-ciocalteu reagent (1:10 dilution with deionized water) and left to stand $2.5 \mathrm{~min}$ at room temperature and then $2 \mathrm{ml}$ of sodium carbonate solution $(7.5 \%$ in deionized water) was added. After incubating $1 \mathrm{~h}$ at room temperature in a dark place the absorbances were measured at $725 \mathrm{~nm}$ by UV spectrophotometer (Perkin Elmer). Results were expressed as milligrams of Gallic acid equivalents (GAE) per gram film weight.

\section{Antibacterial Tests}

The antibacterial properties of films were tested by using disc diffusion method. The film discs with the plant phenolics were applied to the surface of an agar plate containing the microorganism to be tested. The effectiveness of a particular antimicrobial agent is shown by the presence of growth inhibition zones. The zones of inhibition appear as clear areas surrounding the disc from which the substances with antimicrobial activity diffused. All antibacterial tests were performed by using a gram negative (Escherichia coli NRRL B-3008) and a gram positive bacteria (Staphylococcus epidermidis ATCC 12228). Positive control was chosen as penicillin antibiotic disc. After the $24 \mathrm{~h}$ incubation, INT (tetrazolium dye) solution was sprayed over the surface of an agar plate to ensure visible indication of inhibition. INT reacts with the metabolites produced by the microorganisms and the surface with the living microorganisms turn to pink color. For the antibacterial tests of plant extract loaded SF/ CHI blend films, $1900 \mu l$ nutrient broth and $2000 \mu l$ sterilized deionized water were added in each well of 12 well plate and each well inoculated with $200 \mu l$ of $6 \mathrm{~h}$ incubated bacterial suspensions after standardized by adjusting their optical densities at $420 \mathrm{~nm}$ by a UV spectrophotometer to obtain 0.8-1.2 absorbances corresponding to approximately $10^{7} \mathrm{CFU} / \mathrm{ml}$. Sterilized perforated cylindrical plastic cages were placed at the center of each well. This allowed us to put film discs into the well so that turbidity measurements could be performed at the center of each well on the plate. The assay plates were incubated at $37{ }^{\circ} \mathrm{C}$ for $24 \mathrm{~h}$ and the growth kinetic assays for each strain were performed by growth curves and observed as turbidity determined by Varioskan micro-plate reader at $620 \mathrm{~nm}$.

\section{Results and Discussion}

Antibacterial and physical properties of films as wound care products are very important. The products with specific properties can be manufactured by modifying and blending different natural biopolymers to have many advantages over traditional materials. Silk fibroin, which is a natural fibrous protein, has excellent mechanical strength and good biocompatibility, and oxygen and water permeability in the wet state, which suggests that it could be used as a wound 


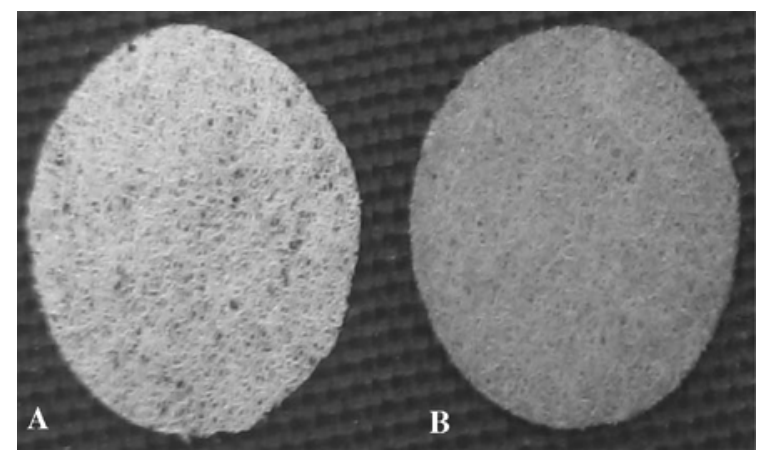

Figure 1. Digital images of $\mathrm{SF} / \mathrm{CHI}$ blend film discs $(10 \mathrm{~mm}$ in diameter); (A) before and (B) after treatment with plant extract solution.

dressing [11,12]. However, the dried as-cast films are brittle and difficult for practical use. A composite structure of SF and $\mathrm{CHI}$ may result in a reinforced structure to be used in biomedical applications.

Digital pictures of $\mathrm{SF} / \mathrm{CHI}$ blend film discs before and after treatment with plant extracts are given in Figure 1(A) and $1(\mathrm{~B})$, respectively. The yellowish color indicates the presence of plant phenolics adsorbed on the surface of films. Specialized additives with special functions can be introduced in advanced wound dressings with the aim to provide strong antibacterial properties. Physical adsorption is a simple method for coating surfaces. It takes advantage of noncovalent interactions such as van der Waals forces, hydrogen bonding, or hydrophobic interactions. Molecules attached through adsorption tend to slowly leach from the surface. Therefore, infection-resistant biomaterials like wool and silk have also been produced by sorption of antibiotics into the material $[18,19]$. It was also reported that the plant phenolics, which possess antibacterial activities, can selectively be adsorbed on the surface of hydrophobic protein SF by hydrophobic interactions [2,5].

The surface and cross-sectional SEM images of SF/CHI blend films are shown in Figure 2(A)-(F). These films consist of fibrous and dense film surfaces. These images show that the fibrous surface consists of randomly arranged SF fibers (10-20 $\mu \mathrm{m}$ in width) which form a network characterized by the presence of many interconnected voids (Figure 1(A)). The network connection of adjacent fibers with thin films can be observed from the SEM image at higher magnification (Figure 2(D)). These features were also observed for the silk fibroin-based formic acid-crosslinked three-dimensional nonwoven material prepared by other researchers [20]. The formation of film between fibers was explained by the good film-forming ability of SF in an acidic solution [20]. In our case, the amount of film observed in the structure of $\mathrm{SF} / \mathrm{CHI}$ blend material was relatively greater than pure SF films. After the evaporation of formic acid, soluble SF and CHI solidified into a thin film which not only connected fibers to each other, but also formed a continuous film structure at the bottom of these fibers (Figure 2(B)).

The surface images of these films (Figure 2(A) and 2(D)) showed a rough surface. The roughness is very evident at high magnification (Figure 2(D)), although the degree of roughness could not be measured by SEM. On the other hand, possibly CHI enriched surfaces of the film exhibited smooth and uniform surfaces, as shown in Figure 2(B) and 2(E). However, some had cracks in a small area of the film (Figure 2(E)), although most of the surface was smooth. This indicated that some physical force was partially applied between the two components at the interface when the biopolymer blend was formed. The cross-sectional images of films are shown in Figure 2(C) and 2(F). The pure SF film did not have a boundary, whereas distinct boundaries of two layers could be observed in SF/CHI blend films. These results indicate that a thin layer of SF and CHI, which have film forming abilities, was maintained during the process of

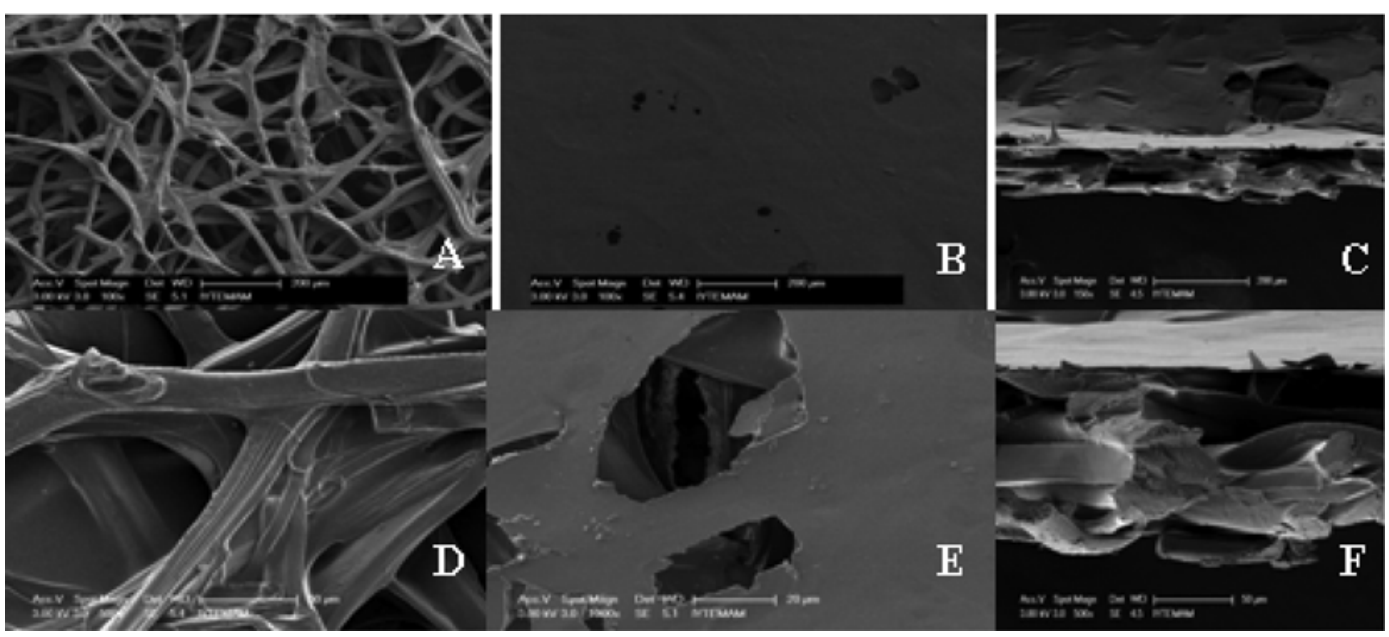

Figure 2. SEM images of the SF/CHI blend film surfaces; (A, D) fibrous surface, (B, E) dense film surface, and (C, F) cross-sectional view. 

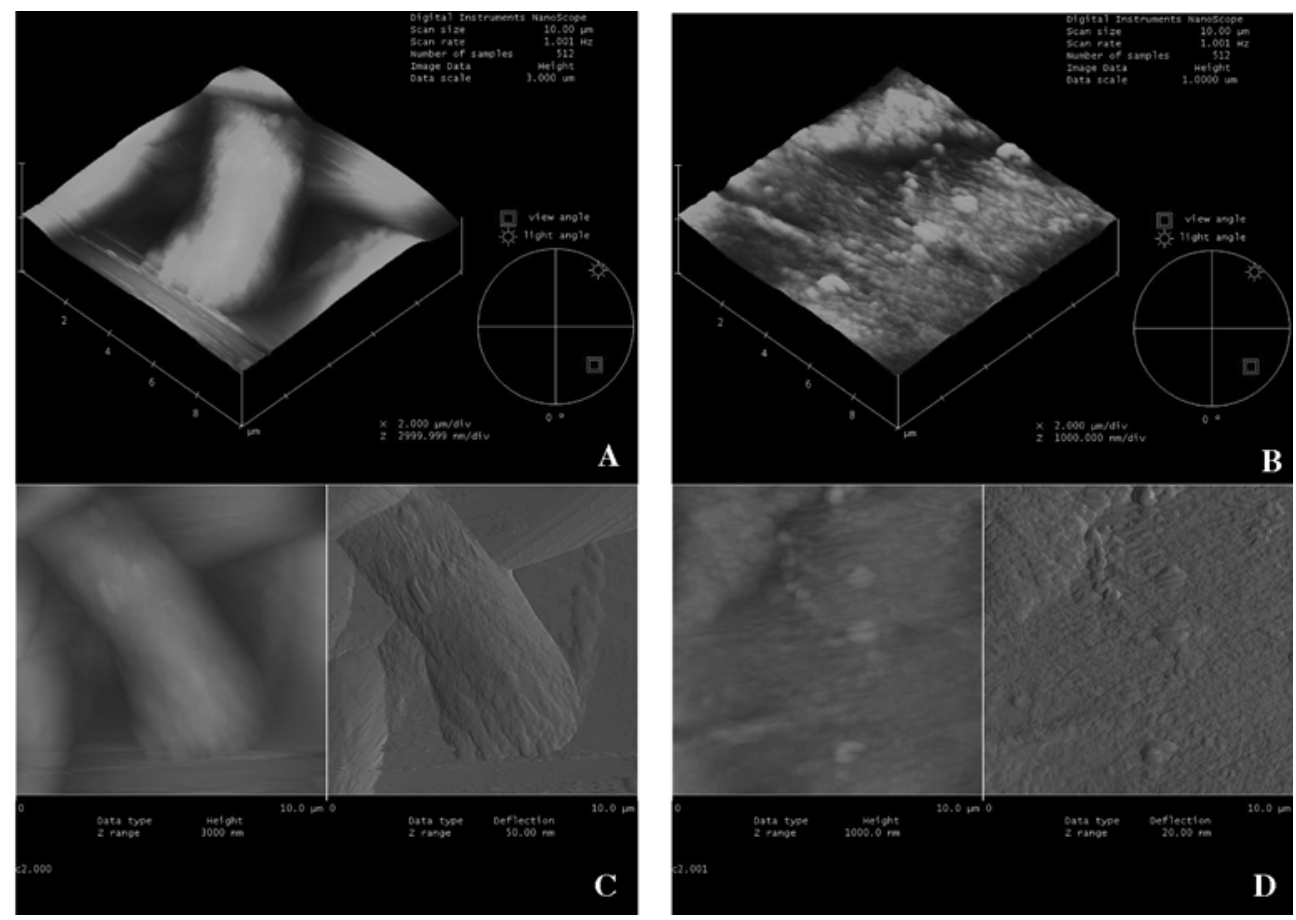

Figure 3. AFM images of SF/CHI film; (A) 3-D images of fibrous surface, (B) dense film surface, (C) the topography of fibrous surface, and (D) dense film surface.

formic acid evaporation. The films prepared in this study can therefore be considered a composite material consisting of $\mathrm{SF}$ fibers incorporated into a matrix of SF and CHI in film form. The similar observations for three-dimensional nonwoven material prepared from SF dissolved in formic acid were alos made by other researhers [20]. It was reported that that the conformational transition of silk fibroin to a $\beta$-sheet structure was induced by chitosan [21,22]. Therefore, both fibers and films are suppossed to be water-insoluble owing to their $\beta$-sheet crystalline structure $[11,12]$. However, differences in features of the two components are expected to have significant effect on the properties of the resulting composite under dry or wet conditions [11,20].

Three-dimensional and topographical AFM images of SF/ $\mathrm{CHI}$ blend film in a scan range of $10 \times 10 \mu \mathrm{m}$ are shown in Figure 3(A), 3(B) and Figure 3(C), 3(D), respectively. The features of both surfaces were in accordance with the SEM observations shown in Figure 2(A)-(F). The surface roughness values were determined as 10.30 and $3.18 \mathrm{~nm}$, for fibrous and smooth film surfaces after treatment with plant extracts, respectively. Treatment with plant extracts did not cause any significant changes in the surface features of these films. A highly rough surfaces were observed for fibrous surfaces due to the presence of fibril structures as shown in Figure 3(A) and 3(C). This feature was also observed in the pure SF film. However, SF/CHI blend film had more developed structure with smooth film and rough fibrous surfaces. The flatness of the film surface is probably attributed to the
Table 1. The total phenol amount adsorbed per gram of film having various blend ratios

\begin{tabular}{lccc}
\hline & \multicolumn{3}{c}{ Total phenol adsorbed $(\mathrm{mg} \mathrm{GAE} / \mathrm{g}$ film $)$} \\
\cline { 2 - 4 } SF (\%) & HE & PT & PL \\
\hline SF100 & 49.70 & 46.05 & 85.27 \\
SF75 & 55.50 & - & - \\
SF50 & 77.15 & 42.75 & 41.37 \\
SF25 & 81.90 & - & - \\
SF00 & 61.30 & 37.05 & 53.50
\end{tabular}

P. terebinthus (PT), P. lentiscus (PL), and H. empetrifolium (HE) plant extracts.

increasing of surface energy while two phases were in contact with the surface of the Petri dish and to their drying process. The observation was consistent with the expectation considered from the SEM results. The surface roughness is assumed to be one of the factors that can affect the bioadhesiveness or cell attachment which coul be very important in biomedical applications.

The fibrous surface of the film exhibits hydrophobic nature and allows the adsorption of phenolics from PT, PL, and HE extract solutions. The total phenol amount adsorbed per gram of film prepared at various blend ratios are tabulated in Table 1. Our results showed that 77.15, 42.75, and $41.37 \mathrm{mg}$ GAE phenolics per gram of SF/CHI blend film (SF50: $50 \%$ SF by weight) could be adsorbed from HE, 


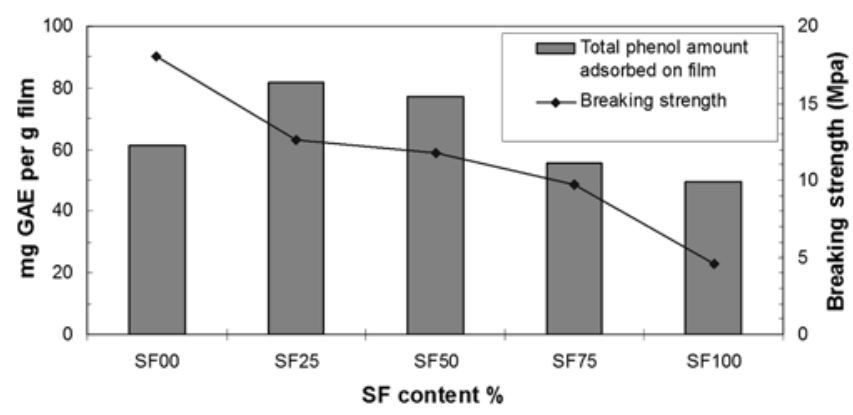

Figure 4. Comparison between the breaking strength of SF/CHI blend films and total phenol amount adsorbed per gram of $\mathrm{SF} / \mathrm{CHI}$ blend film.

PT and PL extract solutions, respectively. SF film alone could adsorb 49.70, 46.05, and 85.27 mg GAE phenolics per gram of film from HE, PT, and PL extract solutions, respectively. CHI film alone could adsorb 61.30, 37.05, and $53.50 \mathrm{mg}$ GAE phenolics per gram of film from HE, PT, and PL extract solutions, respectively.

The effects of the SF/CHI blend ratios (SF content) on the adsorption of phenolics from HE extract solution and mechanical properties were taken into consideration to discover the feasibility of using these films as biomedical materials such as wound dressing. As seen in Figure 4 breaking strength of the SF/CHI blend film decreased with the increase in the SF content. It is well known that the SF film is very brittle in dry conditions. The strength of prepared SF/CHI blend films could be sufficient for practical applications. If needed, a high content of the $\mathrm{CHI}$ component could be used to obtain much stronger film if necessary. The similar observations regarding mechanical properties of $\mathrm{SF} /$ CHI blend films were also reported by other reseachers $[11,12]$. When the adsorption abilities of these blend films were considered, A significant amount of phenolics from HE extract solution was adsorbed by the blend films having SFcontent of 25 to $50 \%$. In the literature, it was also reported that the SF/CHI blend films having 40-50\% chitosan content exhibited very high oxygen permeabilities and good mechanical properties [11]. Therefore, the SF/CHI blend films having at least $25-50 \% \mathrm{SF}$ content can be used as a wound dressing material because of their good phenolic adsorption abilities, good mechanical properties and good water vapor and oxygen permeabilities.

Disc diffusion method is a simple way of determining the susceptibility of a microorganism to an antimicrobial agent by inoculating an agar plate with the culture and allowing the antimicrobial agent to diffuse into the agar medium. As seen in Figure 5, zones of no growth were seen around the disc containing antibacterial properties against the cultured bacteria. Antibacterial test results obtained by disc diffusion method are summarized in Table 2. These inhibition zones were observed around discs of penicillin $(18.0 \mathrm{~mm}$ for $E$. coli; 18.5 for $S$. Epidermidis) and SF/CHI blend films

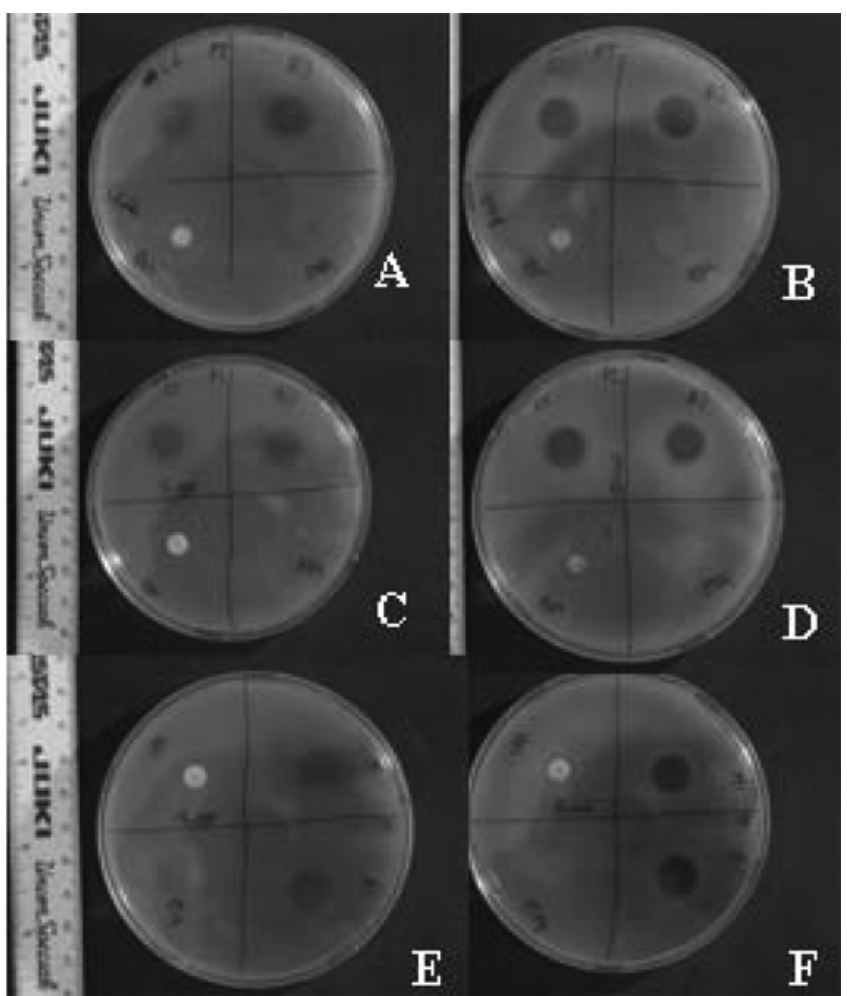

Figure 5. Results of disc diffusion tests against $S$. epidermidis (A, $\mathrm{C}, \mathrm{E})$ and $E$. coli $(\mathrm{B}, \mathrm{D}, \mathrm{F})$ for $\mathrm{SF} / \mathrm{CHI}$ film: C1, B3: with $P$. terebinthus $(\mathrm{PT})$ and P. lentiscus $(\mathrm{PL})$ plant extracts; Eth: without plant extract; Pen: penicillin; A1, B1: with H. empetrifolium $(\mathrm{H})$ plant extract.

Table 2. Antibacterial test results obtained by disc diffusion method

\begin{tabular}{lccccccc}
\hline & \multicolumn{5}{c}{ Inhibition zone diameter (mm) } \\
\cline { 2 - 4 } \cline { 6 - 8 } SF(\%) & PL & PT & HE & & PL & PT & HE \\
\hline SF100 & 18.5 & 14.5 & 13.5 & & 17.5 & 16.0 & 14.0 \\
SF75 & - & - & 13.0 & & - & - & 13.5 \\
SF50 & 13.5 & 12.5 & 14.0 & & 15.0 & 15.0 & 13.0 \\
SF25 & - & - & - & & - & - & 13.0 \\
SF00 & - & - & - & & - & - & 13.0 \\
\hline
\end{tabular}

Inhibition zones for penicillin disc (control): $18.0 \mathrm{~mm}$ for E. coli; $18.5 \mathrm{~mm}$ for S. Epidermidis; P. terebinthus (PT), P.lentiscus (PL), and $H$. empetrifolium (HE) plant extracts.

incorporated with phenolics. The maximum inhibition zones were always observed with pure SF films containing phenolics adsorbed from plant extracts. The inhibition zones observed for $S$. epidermidis were significant compared with those for E. coli. Film discs without plant extracts did not show any inhibition zone and pink color on their surface was the clear indication of bacterial growth.

Turbidity test is the subjective evaluation test carried out 


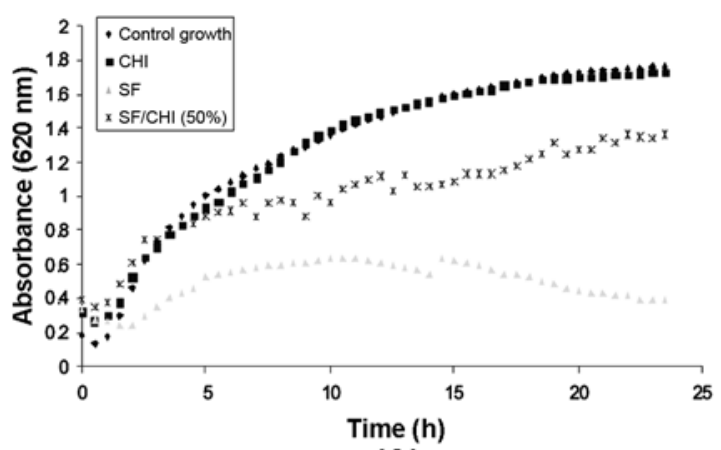

(A)



(C)

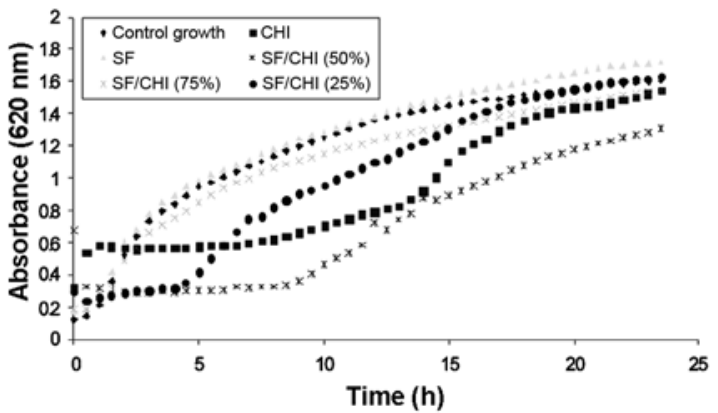

(E)

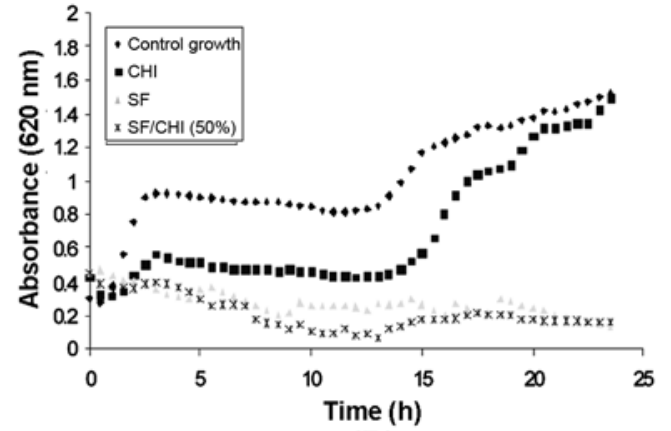

(B)

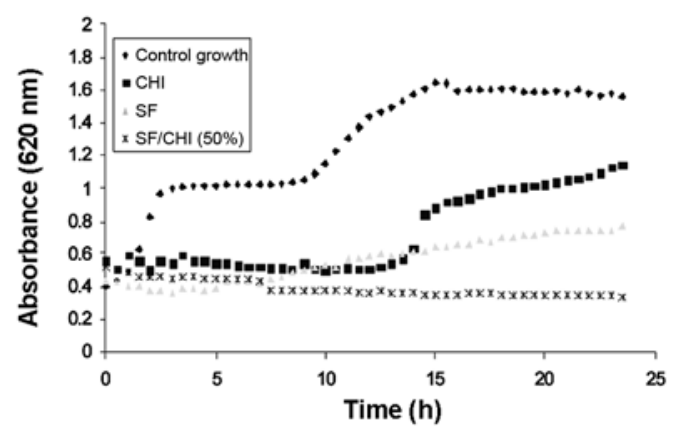

(D)

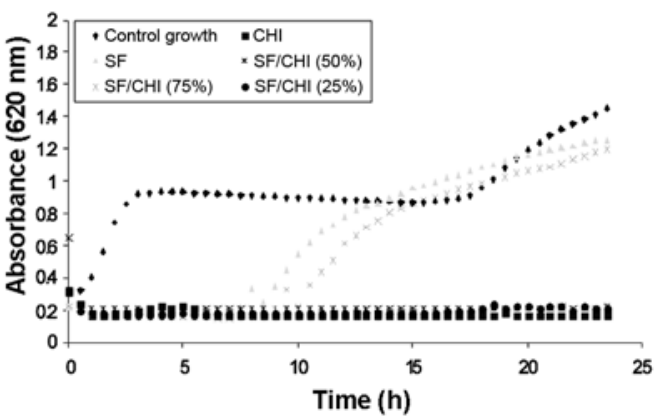

(F)

Figure 6. Results of turbidity tests against E. coli (A, C, E) and S. epidermidis (B, D, F) for SF/CHI film discs loaded with P. terebinthus (A, B); P. lentiscus (C, D); H. empetrifolium (E, F) extracts. SF contents of the films are given in parentheses.

to find the bacterial growth. The result is predicted based on the turbidity of the solution which is determined by the amount of passage of light. The turbidity (absorbance) decreases, as the bacterial growth reduces with the increase in the concentration of the antibacterial agent. Hence, the turbidity rate decreases as the bacterial growth reduces. Results of turbidity tests were in accordance with those obtained from disc diffusion method. As seen from Figure 6(A)-(F), the released antibacterial phenolic compounds from the SF/CHI film discs loaded with PT, PL and HE extracts inhibited the growth of E. coli (Figure 6(A), 6(C), 6(E)) and $S$. epidermidis (Figure 6(B), 6(D), 6(F)). Overall, the plant extract loaded SF/CHI blend films having $50 \% \mathrm{SF}$ content exhibited good antibacterial activities. In some cases, blend films loaded with plant extracts slowed down the growth of bacteria to a certain extent but could not prevent regrowth of bacteria (Figure 6(F)). The effectiveness of antibacterial activity can be attributed to the adsorption and release behaviors of different phenolic compounds originated from different plant extracts having different antibacterial activities. The antibacterial activities observed could be as a result of the antibacterial plant phenolics adsorbed on the surface of hydrophobic protein SF. Similar observations were reported in the literature as well $[2,5]$. Pistacia species are extensively used in folk medicine for the treatment of throat infections and ulcer. It is known that $\alpha$ Tocopherol (vitamin E) is naturally occuring in Pistacia leaves [23]. Pistacia species have antimicrobial potential besides antioxidant and anti-inflammatory activities particularly due to flavonoids, and other phenolic constituents [24]. 
Hypericum species, have been used in different parts of Turkey for their biological activities such as antiseptic, sedative, wound healing, and antibacterial properties. The presence of high phenolic and flavanoid contents of Hypericum species grown in Turkey was reported in the literature [25]. Among different Hypericum species, it was reported that $H$. empetrifolium has the highest antioxidant activity owing to its high flavanoid and phenol content [25]. Therefore, watersoluble phenolics from PT, PL, and HE extracts are good candidates for anitibacterial applications in the form of wound dressing material.

\section{Conclusion}

$\mathrm{SF} / \mathrm{CHI}$ blend films having smooth and fibrous surfaces could play an important role for carrying active antimicrobial and antioxidant compounds which are known to be useful in wound healing. The smooth film surface consisting mainly of chitosan would allow gaseous exchange, act as a barrier to microorganisms, and remove excess exudates from wound site. Therefore, this type of films with plant extract might find a potential application as a wound dressing contact layer material because of unique properties such as high surface area to volume ratio, micro scale fiber diameter, porosity, and light weight.

\section{Acknowledgement}

Authors are grateful for the financial support from Natural Products Research Development Unit (NPRDU/DUAG) located in Izmir Technology Development Zone.

\section{References}

1. Y.-Q. Zhang, Biotechnol. Adv., 16, 961 (1998).

2. D. Baycin, E. Altiok, S. Ulku, and O. Bayraktar, J. Agric. Food Chem., 55, 1127 (2007).

3. Ö. Malay, O. Bayraktar, and A. Batıün, Int. J. Biol. Macromol., 40, 387 (2007).

4. B. B. Pekșen, C. Üzelakçil, A. Güneș, Ö. Malay, and O. Bayraktar, J. Chem. Tech. Biotech., 81, 1218 (2006).

5. E. Altiok, D. Baycin, O. Bayraktar, and S. Ulku, Sep. Pur.
Tech., 62, 342 (2008).

6. Ö. Malay, D. Yalcin, A. Batıgün, and O. Bayraktar, J. Ther. Anal. Calor., 94, 749 (2008).

7. Ö. Malay, O. Bayraktar, Y. Ozgarip, and A. Batıgün, Eur. J. Pharm. Biopharm., 60, 373 (2005).

8. R. Rujiravanit, S. Kruaykitanon, A. M. Jamieson, and S. Tokura, Macromol. Biosci., 3, 604 (2003).

9. L. Meinel, S. Hofmann, V. Karageorgiou, K. H. Carl, J. McCool, and G. Gronowicz, Biomaterials, 26, 147 (2005).

10. J. G. Hardy, L. M. Romer, and T. R. Scheibel, Polymer, 49, 4309 (2008).

11. H. Kweon, H. C. Ha, I. C. Um, and Y. H. Park, J. Appl. Polym. Sci., 80, 928 (2001).

12. J. S. Park, Y. K. Lee, S. W. Ha, and Y. S. Park, J. Appl. Polym. Sci., 74, 2571 (1999).

13. E. S. Sashina, G. Janowska, M. Zaborski, and A. V. Vnuchkin, J. Ther. Anal. Calor., 89, 887 (2007).

14. Y. Ong, J. Wu, S. M. Moochhala, M. H. Tan, and J. Lu, Biomaterials, 29, 4323 (2008).

15. H. Wang, Q. Wei, X. Wang, W. Gao, and X. Zhao, Fiber. Polym., 9, 556 (2008).

16. G. I. Klebanov, O. B. Liubitski, S. E. Ilina, E. O. Medusheva, V. V. Ryltsev, and V. N. Filatov, Biomed. Khim., 52, 69 (2006).

17. A. Y. Mensah, P. J. Houghton, R. A. Dickson, and T. C. Fleischer, Phytother Res., 20, 941 (2006).

18. H. M. Choi, M. Bide, M. Phaneuf, W. Quist, and F. Logerfo, J. Appl. Polym. Sci., 92, 3343 (2004).

19. H. M. Choi, M. Bide, M. Phaneuf, W. Quist, and F. Logerfo, Text. Res. J., 74, 333 (2004).

20. I. D. Pra, A. Chiarini, A. Boschi, G. Freddi, and U. Armato, Int. J. Mol. Med., 18, 241 (2006).

21. X. Chen, W. Li, W. Zhong, Y. Lu, and T. Yu, J. Appl. Polym. Sci., 65, 2257 (1997).

22. X. Chen, W. Li, and T. Yu, J. Polym. Sci. Polym. Phys., 35, 2293 (1997).

23. B. Kivcak and A. Akay, Fitoterapia, 76, 62 (2005).

24. G. Topçu, M. Ay, A. Bilici, C. Sarıkürkçü, M. Öztürk, and A. Ulubelen, Food Chem., 103, 816 (2007).

25. G. E. Meral and S. Konyalıglu, Acta Pharmaceutica Turcica, 45, 183 (2003). 